\title{
Probabilistic spatial power indexes
}

\author{
Stefano Benati · Giuseppe Vittucci Marzetti
}

September 29, 2011

\begin{abstract}
We present a generalization of spatial power indexes able to overcome their main limitations, namely: i) the excessive concentration of power measures; ii) the too high sensitivity to players' location in the ideological space. Voters' propensity to support an issue is modeled via a random utility function with two additive terms: the deterministic term accounts for voters' preference-driven/predictable behavior; the random one is a catch-all term that accounts for all the idiosyncratic/unpredictable factors. The relative strength of the two terms gives rise to a continuum of cases ranging from the Shapley value, where all aggregation patterns are equally probable, to a standard spatial value, like the Owen-Shapley index, where instead the conditional order is fully deterministic. As an illustrative application, we analyze the distribution of power in the Council of Ministers under three different scenarios: i) EU15 Pre-Nice; ii) EU27 Nice Treaty; iii) EU27 Lisbon Treaty.
\end{abstract}

Keywords European Union · Probabilistic values · Owen-Shapley value • Shapley-Shubik index $\cdot$ Spatial power indexes

\section{Introduction}

Decision-making in the European Union (EU) has long received attention among researchers and practitioners, and several studies have applied power indexes to the analysis of the distribution of power between EU members under the different institutional set-ups (e.g. Baldwin and Widgrén 2004; Felsenthal and Machover

Stefano Benati

Department of Sociology, University of Trento, Via Verdi 26, 38122 Trento, Italy

Tel.: +39-0461-282106, Fax: +39-0461-281348

E-mail: stefano.benati@unitn.it

Giuseppe Vittucci Marzetti

Department of Sociology and Social Research, University of Milano-Bicocca,

Via Bicocca degli Arcimboldi 8, 20126 Milan, Italy

Tel.: +39-02-64487457, Fax: +39-02-64487561

E-mail: giuseppe.vittucci@unimib.it 
2001, 2004; Laruelle and Valenciano 2008). This has stimulated a lively debate on the pros and cons of such indexes (e.g. Garrett and Tsebelis 1999b, 2001; Braham and Holler 2005; Napel and Widgrén 2005) and also suggestions on the possible modifications to the original approach needed to overcome its main limitations, namely the lack of consideration of the actors' preferences, of the inter-institutional context, and of the possible strategic behavior of policy makers (see, for instance, Steunenberg et al. 1999; Napel and Widgrén 2004, 2006, 2011).

In this strand are two recent contributions by Barr and Passarelli (2007; 2009), who put forward two generalizations of power indexes to account for both voters' preferences and the role of an agenda-setter, and apply them to the analysis of the distribution of power within the Council of Ministers (CM).

In particular, Barr and Passarelli (2009) present a generalization of the Owen and Shapley (1989) spatial index - which is itself a non-symmetric generalization of the standard Shapley and Shubik (1954) index, made to account for the impact of the ideological location of players and bill contents in the process of coalition formation - to take into account the effect of an agenda-setter on the distribution of power. In the case of CM, the agenda-setter is the European Commission (EC), which has indeed the monopoly of the proposals for the majority of issues.

Although the approach is analytically elegant, it suffers from two major drawbacks: i) it "assigns zero probability to a huge fraction of possible orderings, and this results in excessive concentration of power measures"; ii) "the probability of an alignment does not depend on the distance between two players, but only on the projections of the players' locations on a rotating axis, which measures the political content of the bill; thus, power values are highly sensitive to the positions of the players" (Passarelli and Barr 2007, p.43).

In the paper, we suggest a simple modification of Barr and Passarelli's (2009) index to overcome these limitations. In particular, we apply a random utility model at the dynamics of coalition formation. In this model, players' utilities result from the sum of two components. One is deterministic and depends on the player's propensity to support the issue. The other is random: a catch-all term for all the idiosyncratic and unpredictable factors. The relative importance of the random component over the deterministic one produces a continuum of cases ranging from the Shapley value, in which all the different aggregation patterns are equally probable, to a standard spatial value, like the Owen-Shapley index, where instead the ordering is fully deterministic and preference-driven.

The paper is organized as follows. Section 2 briefly reviews the literature on voting games and power indexes. In Section 3, we discuss our proposed extension. Section 4 shows an application to the analysis of the power distribution among the EU members in the CM, along the lines of Barr and Passarelli (2009). Section 5 concludes.

\section{Voting games and power indexes}

Power indexes measure traditionally the individual probability of being part of or influencing a winning coalition (implementing unspecified policies and with players having no particular preference over outcomes).

The starting point is the definition of a simple game, where, given a set $\mathcal{N}$ of $n$ players, each coalition of players, i.e. each subset $\mathcal{S} \subseteq \mathcal{N}$, is classified as either 
winning or losing. This is conveniently summarized by a characteristic function $v: 2^{\mathcal{N}} \mapsto\{0,1\}$, where $v(\mathcal{S})=1$ if the coalition $\mathcal{S}$ is winning and 0 otherwise.

In voting games, the players are the voters and the mapping $v$ simply summarizes the voting rules. In the standard case of weighted majority games, there is a set of voters-players each controlling $w_{i}$ number of votes (the weight of $i$ ). Players make a yes-or-no decision on some political issue and the issue is passed if the number of supporting votes overcomes a certain threshold $q$. The game's characteristic function is therefore $v(\mathcal{S})=1$ if $\sum_{i \in \mathcal{S}} w_{i} \geq q$ and $v(\mathcal{S})=0$ otherwise.

The most famous power indexes are the Penrose-Banzhaf index (Penrose 1946; Banzhaf 1965) (PBI) - aka Penrose index, absolute Banzhaf index and non normalized Banzhaf index $-{ }^{1}$ and the Shapley-Shubik index (SSI) (Shapley and Shubik 1954). The value attached to each player $i$ by these indexes is just a weighted sum over all possible coalitions not containing $i\left(\mathcal{S}_{i} \subseteq \mathcal{N} \backslash\{i\}\right)$ of $i$ 's marginal contribution:

$$
\phi_{i}(v)=\sum_{\mathcal{S}_{i} \subseteq \mathcal{N} \backslash\{i\}} p\left(\mathcal{S}_{i}\right)\left(v\left(\mathcal{S}_{i} \cup\{i\}\right)-v\left(\mathcal{S}_{i}\right)\right)
$$

with $p\left(\mathcal{S}_{i}\right) \geq 0$.

The marginal contribution of the player $i$ to a coalition $\mathcal{S}_{i}$ is positive (and equal to one) if and only if $i$ is pivotal (or critical or decisive) for the coalition to win, i.e. by joining the coalition, $i$ makes it switch from losing to winning.

The only difference between PBI and SSI is that the former assigns an equal weight to all coalitions $\mathcal{S}_{i}: p\left(\mathcal{S}_{i}\right)=1 / 2^{n-1}$; while the latter gives an equal weight $1 / n$ ! to all the possible orderings of $\mathcal{N}$, so that the probability of a coalition $\mathcal{S}_{i}$ is:

$$
p\left(\mathcal{S}_{i}\right)=\frac{s !(n-s-1) !}{n !}
$$

where $s$ is the cardinality of $\mathcal{S}_{i}$.

SSI can be seen as the application to voting games of the Shapley (1953) value, which, following the axiomatic approach of cooperative game theory, can be proved to be the only value for the games $v$ that satisfies the four axioms of: symmetry, additivity, efficiency, and the dummy player (see Roth 1988). ${ }^{2}$

However, both the indexes can be given an interpretation in terms of expected marginal contribution of player $i$. This is the probabilistic approach, where PBI and SSI are considered cases of a more general class of probabilistic values (Weber 1988), defined by equation $(1)$ with $p\left(\mathcal{S}_{i}\right)$ being the probability attached to the formation of a coalition $\mathcal{S}$ not containing $i$.

This approach makes apparent the process of coalition formation implied by the indexes. As it turns out, PBI and SSI both postulate a completely random process. In particular, PBI results if one assumes that the decisions of the players (different from $i$ ) to join a coalition are all independent random variables with expectation $1 / 2$.

\footnotetext{
1 The Banzhaf index - aka relative or normalized Banzhaf index - is just the Penrose-Banzhaf index normalized so that the index values for all the players sum to one.

2 Einy and Haimanko (2011) have recently shown that, on the domain of simple games, SSI can be uniquely characterized even without the efficiency axiom, which can be replaced by the weaker gain-loss axiom: any power gain by a player must imply a loss for some other player.
} 
The implicit random process behind SSI is instead one in which players randomly join the coalition one by one, and, at the end, all players support the decision (the so-called grand coalition forms). ${ }^{3}$

Both indexes retain the symmetry assumption - the weights do not depend on $i$ - and neglect players' preferences and their possible strategic behavior. This is why, as noted by Napel and Widgrén (2005), the probabilistic assumptions underlying PBI and SSI are basically the Maxwell-Boltzmann statistics and the Bose-Einstein statistics used in physics to model the behavior of particles.

These assumptions can be defended. As for the lack of strategic considerations, one can argue that not all political decisions are taken strategically. Indeed, strategic reasoning can hardly be a secure guide of action when political preferences are not unidimensional or there are thousands of different players (as in the case of a referendum involving millions of voters).

As for the non consideration of players' location over the political space, the assumption is usually defended from an a-priori perspective, where the "principle of insufficient reason" is invoked and the analysis is intentionally carried out from behind a veil-of-ignorance.

A different perspective is adopted in the theory of spatial indexes, where players' preferences over political outcomes enter explicitly the calculation of the index. ${ }^{4}$ This is the case of the Owen-Shapley index (Owen and Shapley 1989), a nonsymmetric generalization of SSI.

Building on Owen's (1971) intuition, Owen and Shapley (1989) postulate a process of coalition formation determined by the ideological distance between voters in the political space. In particular, given the set of players' ideal (or bliss) points $\lambda_{i} \in \mathcal{R}^{m}$, defined in a $m$-dimensional Euclidean space, where each dimension captures an ideological parameter of the game, each non-zero vector $\theta \in \Theta \subseteq \mathcal{R}^{m}$ is assumed to induce an ordering $\prec$ on $\mathcal{N}$ :

$$
j \prec i \Leftrightarrow u\left(\lambda_{j}, \theta\right)>u\left(\lambda_{i}, \theta\right)
$$

i.e. player $j$ precedes $i$ if and only if $u\left(\lambda_{j}, \theta\right)>u\left(\lambda_{i}, \theta\right)$.

Given players' bliss points $(\lambda)$ and the bill content $(\theta)$, the ordering in which the players join the coalition is therefore fully determined by the function $u\left(\lambda_{i}, \theta\right)$, which measures the "enthusiasm" of each player for the issue.

When $\theta$ is a stochastic vector drawn from a probability distribution $F_{\theta}: \Theta \mapsto$ $[0,1]$ with continuous density function $f_{\theta}(\theta)$, the probability of observing a certain coalition $\mathcal{S}_{i}$ is thus:

$$
p\left(\mathcal{S}_{i}\right)=\int \cdots \int_{A\left(\mathcal{S}_{i}, \theta\right)} f_{\theta}(\theta) \mathrm{d} \theta
$$

\footnotetext{
3 Alternatively, one can derive SSI from independent Bernoulli experiments when the joint probabilities of all the players different from $i$ are identical and drawn from a uniform distribution on $[0,1]$ (Straffin 1977).

4 There has been a lively debate on the possibility and utility of taking preferences into power measurements. To do so, thus overcoming some of the limitations emphasized by Garrett and Tsebelis (1999b), Steunenberg et al. (1999) define and measure power as the expected relative distance between the equilibrium outcome and the player's ideal point. The approach is strongly criticized, among the others, by Braham and Holler (2005), who argue about the theoretical senselessness of preference-based power indexes. To respond to such critique and clearly distinguish between power and success, Napel and Widgrén (2004) propose to measure (ex-post) power as the sensitivity of the expected or observed outcome to individual players' actions or preferences. The approach is developed further in Napel and Widgrén (2006, 2011).
} 
where $A\left(\mathcal{S}_{i}, \theta\right) \subseteq \Theta$ is a correspondence that identifies the subset of issues consistent with the coalition $\mathcal{S}_{i}$, that is such that $u\left(\lambda_{j}, \theta\right)>u\left(\lambda_{i}, \theta\right)$ if and only if $j \in \mathcal{S}_{i}$.

Owen and Shapley (1989) assume that $\theta$ is randomly drawn from the unit-sphere $H_{m-1}$ by a uniform distribution and that the function $u$ is the inner product of $\lambda_{i}$ and $\theta:^{5}$

$$
u\left(\lambda_{i}, \theta\right)=\left\langle\lambda_{i}, \theta\right\rangle
$$

As noted by Barr and Passarelli (2009), equation (4) can define a more general class of indexes, that they call probabilistic spatial values. Indeed, given a probability distribution over issues $F_{\theta}(\theta)$ and an ordering function $u\left(\lambda_{i}, \theta\right)$, it is always possible via equations (4) and (1) to characterize a probabilistic value in a spatial context that satisfies the efficiency axiom, i.e. $\sum_{i \in \mathcal{N}} \phi_{i}(v)=v(\mathcal{N})$.

They also consider the role of an agenda-setter in influencing players' power via the determination of the content of the proposal. In particular, they assume that the bill actually proposed is unequivocally determined by the agenda-setter and what generates players' uncertainty is only the limited knowledge about the agenda-setter type. Hence, given the probability distribution of the agenda type and a one-to-one relation between the agenda type and the bill, the implied probabilistic spatial values can be computed.

What makes the approach appealing is that the orderings of players are generated by random political issues and players are close in a certain order because they share similar views on the policy issue. Its main drawbacks are that: i) it "assigns zero probability to a huge fraction of possible orderings, and this results in excessive concentration of power measures"; ii) "the probability of an alignment does not depend on the distance between two players, but only on the projections of the players' locations on a rotating axis, which measures the political content of the bill; thus, power values are highly sensitive to the positions of the players" (Passarelli and Barr 2007, p.43).

In the next section we discuss a simple generalization that is able to overcome these limitations. In particular, we apply a random utility model to the dynamics of coalition formation, thus adding a random component to the bill-induced ordering of players. The relative strength of this component produces a continuum of cases ranging from the deterministic order (conditional on the issue) of equation (4) and the fully random order of equation (2).

\section{A random utility model of coalition formation}

Let $\Theta$ be a normed vector space, subset of a $m$-dimensional Euclidean political space $\left(\Theta \subseteq \mathcal{R}^{m}\right)$. $\Theta$ represents the set of all the issues to vote on. Each issue is represented by a vector $\theta \in \Theta$. Let $\tilde{u}_{i}(\theta)$ be a random variable measuring the utility player $i$ gets from $\theta$ (or equivalently the "enthusiasm" of $i$ toward $\theta$ ):

$$
\tilde{u}_{i}(\theta)=U_{i}(\theta)+\tilde{\epsilon}_{i}
$$

5 Owen and Shapley (1989) prove that the spatial values so defined are related to the so called Copeland winner (or strong point), i.e. the policy outcome with the lowest probability to be beaten by any other alternative. In particular, they prove that, in a bi-dimensional ideological space, the strong point is simply a linear combination of players' ideal points with coefficients equal to the Owen-Shapley indexes: $\sum_{i \in \mathcal{N}} \phi_{i} \lambda_{i}$. Unfortunately, this elegant result does hold neither for preferences defined in political spaces of three or more dimensions, nor for non-decisive games, i.e. voting games in which $\exists \mathcal{S}: v(\mathcal{S})=0 \wedge v(\mathcal{N} \backslash \mathcal{S})=0$. 
where $U_{i}: \Theta \mapsto \mathcal{R}$ is a real valued function and $\tilde{\epsilon}_{i}$ a random variable with probability distribution $F_{\epsilon}\left(\epsilon_{i}\right)$ and continuous density function $f_{\epsilon}\left(\epsilon_{i}\right)$.

The total utility is therefore modeled as the sum of: i) a deterministic component capturing the predictable utility player $i$ gets from the issue; ii) a random component modeling the idiosyncratic and unpredictable behavior of the player. This component includes unobservable features of $\theta$ and contingent political behavior of $i$, to be considered largely unpredictable. When the deterministic component prevails, $i$ 's behavior is by and large predictable. Conversely, when the random component prevails, $i$ 's behavior is mostly erratic and unpredictable, although still describable in terms of probabilities. ${ }^{6,7}$

As in Owen and Shapley (1989), we assume that via $u$, each $\theta$ induces an ordering $\prec$ on $\mathcal{N}$ :

$$
j \prec i \Leftrightarrow u_{j}(\theta)>u_{i}(\theta)
$$

i.e. player $j$ precedes $i$ if and only if $u_{j}(\theta)>u_{i}(\theta)$, where differences between $u_{i}$ and $u_{j}$ could reflect, for instance, different ideological positions of $i$ and $j$ in the policy space $\Theta$.

Given the ordering $\prec$, we say that a coalition $\mathcal{S}_{i} \subseteq \mathcal{N} \backslash\{i\}$ - i.e. not containing $i$ - is consistent with $\prec$ if and only if all the players who (do not) belong to the coalition (do not) precede player $i$ according to $\prec$.

Thus, the probability (conditional on $\theta$ ) that the coalition $\mathcal{S}_{i}$ is consistent with the ordering $\prec$ that results from the realizations of the random utilities $\tilde{u}$ is:

$$
\begin{aligned}
p\left(\mathcal{S}_{i} \mid \theta\right)= & \operatorname{Pr}\left(\left(\tilde{u}_{j}(\theta)>\tilde{u}_{i}(\theta), \forall j \in \mathcal{S}_{i}\right) \cap\left(\tilde{u}_{j}(\theta)<\tilde{u}_{i}(\theta), \forall j \in \mathcal{N} \backslash \mathcal{S}_{i} \backslash\{i\}\right) \mid \theta\right) \\
= & \int_{-\infty}^{+\infty}\left(\prod_{j \in \mathcal{S}_{i}}\left(1-F_{\epsilon}\left(\epsilon_{i}+U_{i}(\theta)-U_{j}(\theta)\right)\right)\right) . \\
& \cdot\left(\prod_{j \in \mathcal{N} \backslash \mathcal{S}_{i} \backslash\{i\}} F_{\epsilon}\left(\epsilon_{i}+U_{i}(\theta)-U_{j}(\theta)\right)\right) f_{\epsilon}\left(\epsilon_{i}\right) \mathrm{d} \epsilon_{i}
\end{aligned}
$$

6 As stressed by Napel and Widgrén (2005, 2011), in case of a supermajority rule, the policy outcome is also affected by the location of the status quo. Hence, equation (6) strictly applies only when the status quo lies outside voters' Pareto set. It is however possible to introduce the legislative status quo $q \in \mathcal{Q} \subset \mathcal{R}^{m}$ among the determinants of the "enthusiasm" of the players towards the proposal $\theta$. In this case the enthusiasm function could be expressed as:

$$
\tilde{u}_{i}(\theta, q)=U_{i}(\theta)-U_{i}(q)+\tilde{\epsilon}_{i}
$$

where $U_{i}$ is intended like before as the utility player $i$ gets from the outcome.

7 Napel and Widgrén (2008) have stressed the possibility of giving SSI a more explicit spatial interpretation. Indeed, SSI can be conceived as the probability of being in the median position (and so the Condorcet winner in unidimensional political spaces with single-peaked preferences) when the players' bliss points are drawn uniformly at random. Our idea of local random perturbations can also be applied to this setting. Indeed, one can assume that, instead of dealing with fixed and constant bliss points (and so policy outcomes fully determined by the median) or bliss points drawn uniformly at random, players' bliss points can have both a constant and a random component:

$$
\tilde{\lambda}_{i}=\lambda_{i}+\tilde{\epsilon}_{i}
$$

This is very similar to what done by Passarelli and Barr (2007). 
Equation (9) can be interpreted also in terms of random sequences. Indeed, let $O R$ be the set of all one-to-one functions $\tau: \mathcal{N} \mapsto\{1, \ldots, n\}$. Each $\tau$ is thus identified with a complete order on $\mathcal{N}$. Moreover, let $\mathcal{P}_{\mathcal{S}_{i}}=\left\{\tau \in O R: \tau(j)<\tau(i)\right.$ iff $\left.j \in \mathcal{S}_{i}\right\}$ denote the set of all the orders in which all and only the players in $\mathcal{S}_{i}$ come before $i$. Finally, let $\operatorname{Pr}(\tilde{\tau}=\tau \mid \theta)$ be the probability of observing a sequence $\tau$ conditional on $\theta$. The following can be proved:

Lemma $1 p\left(\mathcal{S}_{i} \mid \theta\right)=\sum_{\tau \in \mathcal{P}_{\mathcal{S}_{i}}} \operatorname{Pr}(\tilde{\tau}=\tau \mid \theta)$.

Proof A sequence $\tau$ is in $\mathcal{P}_{\mathcal{S}_{i}}$ if and only if all utilities $\tilde{u}_{j}>\tilde{u}_{i}$ for all $\tau(j)<\tau(i)$ and $\tilde{u}_{j}<\tilde{u}_{i}$ for all $\tau(j)>\tau(i)$.

If $\theta$ is a stochastic vector with continuous density function $f_{\theta}(\theta)$, the unconditional probability that the coalition $\mathcal{S}_{i}$ is consistent with the realized ordering $\prec$ is:

$$
p\left(\mathcal{S}_{i}\right)=\int \cdots \int_{\Theta} p\left(\mathcal{S}_{i} \mid \theta\right) f_{\theta}(\theta) \mathrm{d} \theta
$$

By plugging equation (10) into (1), one obtains a probabilistic generalized spatial value (PGSV), which is a particular class of probabilistic values, since, for a given simple game $v$, player $i$ 's value can be seen as the probability of being in a pivotal position, out of all the possible coalitions that the random issues and utilities can produce. ${ }^{8}$

It is possible to prove that any PGSV may represent a distribution scheme of the full yield of the game, i.e. it satisfies the efficiency axiom: $\sum_{i \in \mathcal{N}} \phi_{i}(v)=v(\mathcal{N})$.

Proposition 1 Any PGSV satisfies the efficiency axiom.

To prove efficiency it is sufficient to prove that PGSV is a random-order value, i.e. a probabilistic value that admits a random-order description, since, as proved in Weber (1988), a probabilistic value is a random order value if and only if it is a quasivalue, i.e. an efficient probabilistic value.

Lemma 2 Any PGSV is a random-order value.

Proof Let $D_{i}(v, \tau)$ be the marginal contribution of $i$ to the coalition of players that precede him according to $\tau$, i.e.:

$$
D_{i}(v, \tau)=v(\{j \in \mathcal{N}: \tau(j) \leq \tau(i)\})-v(\{j \in \mathcal{N}: \tau(j)<\tau(i)\})
$$

It can be verified that, for each $\tau \in \mathcal{P}_{\mathcal{S}_{i}}, D_{i}(v, \tau)=v\left(\mathcal{S}_{i} \cup\{i\}\right)-v\left(\mathcal{S}_{i}\right)$. Moreover, from Lemma $1, p\left(\mathcal{S}_{i} \mid \theta\right)=\sum_{\tau \in \mathcal{P}_{\mathcal{S}_{i}}} \operatorname{Pr}(\tilde{\tau}=\tau \mid \theta)$. It follows that:

$$
\left(v\left(\mathcal{S}_{i} \cup\{i\}\right)-v\left(\mathcal{S}_{i}\right)\right) p\left(\mathcal{S}_{i} \mid \theta\right)=\sum_{\tau \in \mathcal{P}_{\mathcal{S}_{i}}} D_{i}(v, \tau) \operatorname{Pr}(\tilde{\tau}=\tau \mid \theta)
$$

\footnotetext{
8 If one considers the possible effect of the status quo $q$ (equation 7 ) and assume it is a stochastic vector with continuous density function, the probability in equation (9) should be intended as conditional on $\theta$ and $q$. To recover the unconditional probability one must simply integrate over $\theta$ and $q$. None of the subsequent results actually changes.
} 
PGSV of player $i$ is therefore:

$$
\begin{aligned}
\phi_{i}(v) & =\int \cdots \int_{\Theta} \sum_{\mathcal{S}_{i} \subseteq \mathcal{N} \backslash\{i\}}\left(v\left(\mathcal{S}_{i} \cup\{i\}\right)-v\left(\mathcal{S}_{i}\right)\right) p\left(\mathcal{S}_{i} \mid \theta\right) f_{\theta}(\theta) \mathrm{d} \theta \\
& =\int \cdots \int_{\Theta} \sum_{\mathcal{S}_{i} \subseteq \mathcal{N} \backslash\{i\}} \sum_{\tau \in \mathcal{P}_{\mathcal{S}_{i}}} D_{i}(v, \tau) \operatorname{Pr}(\tilde{\tau}=\tau \mid \theta) f_{\theta}(\theta) \mathrm{d} \theta \\
& =\sum_{\tau \in O R} D_{i}(v, \tau) \operatorname{Pr}(\tilde{\tau}=\tau)
\end{aligned}
$$

which corresponds to the definition of random-order value (see, for instance, Monderer and Samet 2002).

Thus, one can always give a random-order characterization of PGSV. Moreover, when there is no difference between players' preferences over issues, the following can be proved:

Proposition 2 If $U_{i} \equiv U$ for every player, PGSV coincides with the Shapley value.

To prove this proposition it is sufficient to show that in this case PGSV is also a semivalue, i.e. a symmetric probabilistic value, since, as proved by Weber (1988), the Shapley value is the unique symmetric quasivalue.

Lemma 3 If $U_{i} \equiv U$ for every player, then PGSV is a semivalue.

Proof When $U_{i} \equiv U$ for all $i \in \mathcal{N}$, equation (9) is equal to:

$$
p\left(\mathcal{S}_{i} \mid \theta\right)=\int_{0}^{1}\left(1-F_{\epsilon}\left(\epsilon_{i}\right)\right)^{s} F_{\epsilon}\left(\epsilon_{i}\right)^{n-s-1} \mathrm{~d} F_{\epsilon}\left(\epsilon_{i}\right)
$$

where $s=\left|\mathcal{S}_{i}\right|$. Hence, $p\left(\mathcal{S}_{i} \mid \theta\right)=p\left(\mathcal{S}_{i}\right)$ and PGSV can be written as:

$$
\phi_{i}(v)=\sum_{\mathcal{S}_{i} \subseteq \mathcal{N} \backslash\{i\}} \beta^{n}(s)\left(v\left(\mathcal{S}_{i} \cup\{i\}\right)-v\left(\mathcal{S}_{i}\right)\right)
$$

with

$$
\beta^{n}(s)=\int_{0}^{1} t^{s}(1-t)^{n-s-1} \mathrm{~d} t
$$

where $t=1-F_{\epsilon}\left(\epsilon_{i}\right)$ is a Borel probability measure on $[0,1]$. As proved by Dubey et al. (1981), the existence of this measure is a sufficient (and necessary) condition for the value to be a probabilistic symmetric value (see Theorem 11 in Monderer and Samet 2002, p. 2068).

While PGSV converges to the Shapley value when the deterministic impacts of the different issues on players' utility tend to be equal, it instead becomes a probabilistic spatial value (Barr and Passarelli 2009) when the random component disappears. Indeed, in this case $p\left(\mathcal{S}_{i} \mid \theta\right)=1$ if $U_{j}(\theta)>U_{i}(\theta)$ for each and only $j \in \mathcal{S}_{i}$, and 0 otherwise. Hence, equation (10) can be rewritten as (4).

So far, we have said nothing about the actual form of the functions $U_{i}(\theta)$. By assuming single-peaked preferences of players (with bliss point $\lambda_{i} \in \mathcal{R}^{m}$ ), one possibility is to apply equation (5), i.e. define $u(\cdot)$ as the inner product, and restrict $\theta$ to lie in the unit sphere, like done by Owen and Shapley (1989) and Barr and Passarelli (2009). 
Indeed, as proved by Owen and Shapley (1989), in case of bi-dimensional political spaces and uniform distribution of $\theta$, this solution has a very nice relation with the Copeland winner (see footnote 5 ). However, such property does not hold for spaces with more than two dimensions; neither does it hold when the distribution of the issues is not uniform, as in Barr and Passarelli (2009).

Hence, we use a more standard form of players' utility (i.e. the "enthusiasm" function) by assuming Euclidean preferences, as it is usual in spatial models of collective choice, ${ }^{9}$ and we do not restrict the content of the proposal within the unit sphere.

We therefore specify equation (6) as follows:

$$
\tilde{u}_{i}(\theta)=-\rho\left\|\lambda_{i}-\theta\right\|+\tilde{\epsilon}_{i}
$$

where $\|$.$\| is the Euclidean norm and \rho(\geq 0)$ is a coefficient of spatial friction, which describes how players discount distance. ${ }^{10}$

In equation (11) the utility is assumed to decrease linearly with distance at the rate $\rho$, so that, the smaller the distance, the higher the value the player attaches to the possible outcome. When $\rho=0$, players do not care about the actual content of the bill, so their behavior is random and PGSV coincides with the Shapley value. On the contrary, when $\rho \rightarrow+\infty$, players are strongly committed to their ideal points and less disposed to move away from them. Therefore, PSGV tends to a probabilistic spatial value, in which randomness is only on the potential positions of $\theta$.

As for the random component, in line with the standard literature on random utility models, we assume a general extreme value distribution, which gives rise in that literature to the class of logit models (McFadden 1984; Ben-Akiva and Lerman 1985). In particular, we assume that each random term has the following distribution:

$$
F\left(\epsilon_{i}\right)=\mathrm{e}^{-\mathrm{e}^{-\epsilon_{i}}}
$$

The random components are therefore independent and identically Gumbel (or type I extreme value) distributed with location parameter 0 and scale parameter 1.11

9 A critical analysis of this characterization is given by Milyo (2000). Psychologists and cognitive scientists usually assume that the perceived similarity exponentially decays with distance (e.g. Shepard 1987). Humphreys and Laver (2010) analyze instead the case of "city block" preferences, showing that in this case the dimension-by-dimension median can be a majority-rule equilibrium. One can easily incorporate this assumption in our framework by substituting the Euclidean norm with the Manhattan norm in equation (11).

10 In the specification with the status quo, equation (7) becomes:

$$
\tilde{u}_{i}(\theta, q)=-\rho\left(\left\|\lambda_{i}-\theta\right\|-\left\|\lambda_{i}-q\right\|\right)+\tilde{\epsilon}_{i}
$$

11 The assumption that the disturbances are Gumbel distributed is made just for analytical convenience, but it can be defended as an approximation to the normal density (see, for instance, Ben-Akiva and Lerman 1985, Ch. 5). For an analytical survey on random utility models and the presentation of a generalized version able to integrate all the different variants see Walker and Ben-Akiva (2002). 
With this assumption, equation (9) becomes:

$$
\begin{aligned}
p\left(\mathcal{S}_{i} \mid \theta\right) & =\int_{-\infty}^{+\infty}\left(\prod_{j \in \mathcal{S}_{i}}\left(1-\mathrm{e}^{-\mathrm{e}^{-\epsilon_{i}-\rho\left\|\lambda_{j}-\theta\right\|}}\right)\right) . \\
& \cdot\left(\mathrm{e}^{-\mathrm{e}^{-\epsilon_{i}} \sum_{j \in \mathcal{N} \backslash \mathcal{S}_{i} \backslash\{i\}} \mathrm{e}^{-\rho\left\|\lambda_{j}-\theta\right\|}}\right) \cdot \mathrm{e}^{-\mathrm{e}^{-\epsilon_{i}-\rho\left\|\lambda_{i}-\theta\right\|}-\epsilon_{i}-\rho\left\|\lambda_{i}-\theta\right\|} \mathrm{d} \epsilon_{i}
\end{aligned}
$$

As a consequence, in the random-order description of PGSV, the probability (conditional on the issue $\theta$ ) to be the $s+1$-th player in a sequence $\tau$ where the first $s$ players belong to $\mathcal{S}_{i}$ is:

$$
\operatorname{Pr}\left(\tilde{u}_{i}(\theta)>\tilde{u}_{j}(\theta), \forall j \in \mathcal{N} \backslash \mathcal{S}_{i} \backslash\{i\}\right)=\frac{\mathrm{e}^{-\rho\left\|\lambda_{i}-\theta\right\|}}{\sum_{j \in \mathcal{N} \backslash \mathcal{S}_{i}} \mathrm{e}^{-\rho\left\|\lambda_{j}-\theta\right\|}}
$$

where the equality is a standard result of multinomial logit models (see, for instance, McFadden 1984). Formula (15) turns out useful when the process of coalition formation is simulated.

In the next section, we carry out an illustrative application to the standard analysis of the power distribution within the Council of Ministers. The computational aspects and the algorithms used to calculate the indexes are discussed in the Appendix.

\section{Application to the Council of Ministers}

As an illustration of our index, along the lines of Barr and Passarelli (2009) we carry out an analysis of power in the Council of Ministers (CM) under three different scenarios: i) the Pre-Nice scenario: 15 member states and a qualified majority system with a vote allocation less favorable to big countries; ii) the Post-Nice scenario after the enlargement: 27 member states with a re-allocation of the number of votes; iii) the Lisbon Treaty scenario: 27 member states and a substantial qualified double majority system of member states and population.

Countries' ideal points are represented on a bi-dimensional political space. The data are taken from Barr and Passarelli (2009) to increase the comparability with the results of their analysis (Figure 1). ${ }^{12}$

\footnotetext{
12 Barr and Passarelli (2009) compute the points by using the Eurobarometer surveys (Fall 2001-Spring 2003). They employ factor analysis and show that two factors can account for over $70 \%$ of the total variance. Furthermore, they apply the varimax rotation procedure to these factors and inspect the rotated factor loadings, thus finding that the first factor is highly correlated with inter-national issues, while the second is associated with intra-national ones. Finally, for each country they compute the factor scores (reported in Figure 1). The horizontal axes in the Figures are therefore supposed to measure the countries' willingness of a strong EU on the international scene (inter-national stance), whereas the vertical axes are assumed to measure the desired degree of EU involvement in the domestic policies of members (intranational stance). By construction, these scores have zero mean and unit standard deviation. Hence, each point identifies relative attitudes of the countries toward the EU and "the origin of the graph somehow reflects the barycenter of the political space" (Barr and Passarelli 2009, p. 351). That is why the enlargement, which is an institutional change, entails a modification of the countries' position, although it does not imply that the member states change their stances toward the EU. Hence, following Barr and Passarelli (2009), we use the points in Figure 1(a) for the Pre-Nice EU15 scenario and the points showed in Figure 1(b) for the other scenarios.
} 


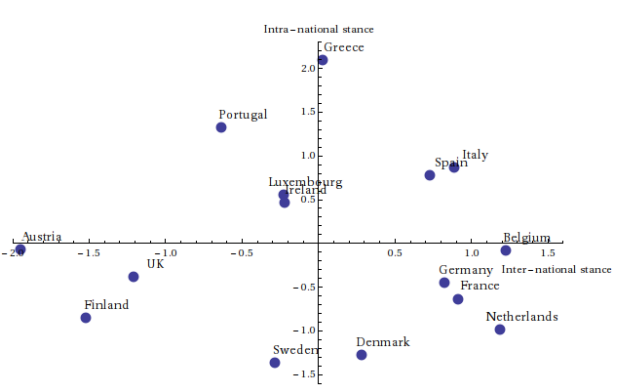

(a) EU15

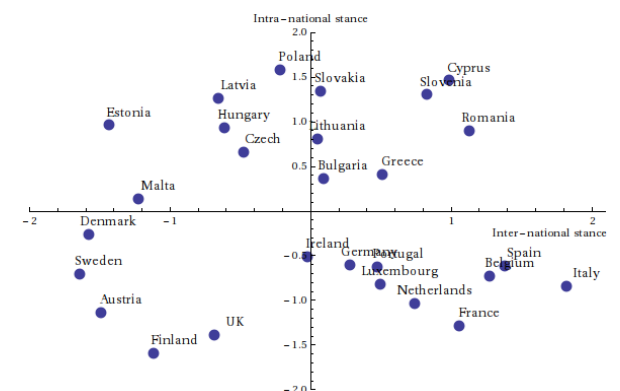

(b) EU27

Fig. 1 EU members' ideal points on a bi-dimensional policy space

Since in PGSV the relative strength of the deterministic component is crucial in driving the result, instead of revolving around an arbitrarily chosen weight, we present each result for a range of possible values. In particular, we compute each index for increasing values of the spatial friction coefficient $\rho$ of equation (11), so moving from SSI to a probabilistic spatial index. This can be taken also as a check of the robustness of a standard spatial index.

Because power in spatial indexes strongly depends on the actual content of the proposal, we first carry out the analysis for a constant issue and then assume a probabilistic distribution over issues. In this respect, in spite of the fact that it is the European Commission (EC) to determine the content of the proposal for the majority of issues, while CM can only accept or reject them, ${ }^{13}$ we leave aside in our illustrative application the agenda-setting role of the Commission. We take instead the countries' internal perspective and assume that the content of the proposal always corresponds to the bliss point of one of the member states.

At a theoretical level, this can be justified by noting that the Presidency of the Council rotates every six months between the governments, and the President sets the daily agenda. Before the Lisbon Treaty, this coordination power was very high because the member country in charge of the Presidency of the CM was also the President of the European Council. ${ }^{14}$

At a practical level, the assumption allows us to compute the index with a meaningful and simple uniform discrete distribution for the issue. This strongly reduces the computational burden. We can thus compute the index in the PreNice scenario with a quasi exact algorithm (Algorithm 2 in the Appendix). This notwithstanding, because of the large number of member countries after the EU

\footnotetext{
13 Two clarifications are in order. First, the Council can always amend the EC proposal by unanimity. Second, the consultation procedure, introduced in the Treaty of Rome (1957), is going to be very much replaced by the codecision procedure, introduced by the Treaty of Maastricht (1991) and called ordinary legislative procedure in the Lisbon Treaty. In their recent contribution, Napel and Widgrén (2011) also consider the possibility of CM to amend the proposal in the consultation procedure. Napel and Widgrén (2006) instead analyze the distribution of power between the EC and the European Parliament in the codecision procedure. 14 The Lisbon Treaty has now reduced its role by officially separating CM and European Council. Moreover, in order to increase continuity in political programs, it is now practice that the three successive presidencies cooperate for a period of 18 months. These are the so-called presidency trios.
} 
enlargement, in the Post-Nice and Lisbon Treaty scenarios we must still rely on Monte Carlo simulations for the computation (Algorithm 1 in the Appendix).

The analysis is meant to be just an illustration of the index we propose and the results are in fact subject to a number of important caveats. First, they crucially depend on the particular functional form of the preferences and the distribution of the issues we have assumed. Second, we use the citizens' preferences to proxy the preferences of their elected representatives, and this is not necessarily true because principal-agent problems can arise. Third, in order to increase the comparability with Barr and Passarelli's (2009) analysis, we employ their data. Nonetheless, countries' ideal points in these data are estimated by means of factor analysis and this kind of analysis usually implies a great loss of information and some (not always transparent) data-mining. Moreover, the data are not recent and preferences can change in time. What is more, there have been likely significant changes since the recent financial crisis and the present Europe debt crisis, and those changes are not captured by these data. Fourth, we have intentionally left out from the analysis all the strategic considerations, but in reality they could matter: countries can strategically commit to political positions to maximize their power, and in repeated voting games there can be cross-issue compromising and logrolling.

With these caveats in mind, in what follows we briefly present and discuss some of the results. ${ }^{15}$

\subsection{EU15 Pre-Nice}

For the Pre-Nice scenario, in order to better disentangle the different mechanisms at work, we take as a case in point Germany and show: i) countries' indexes when the issue to vote coincides with Germany's bliss point; ii) Germany's power when the content of the proposal coincides with the bliss point of the other countries.

In particular, Figure 2 shows the power of six countries - namely, Finland, France, Italy, Portugal, Spain and the UK - for a range of possible values of the spatial friction coefficient $(0 \leq \rho \leq 5)$ when the proposal coincides with Germany's bliss point. In this case, given our specification of the enthusiasm function (equation 11), the players' locations in the ideological space (Figure 1(a)) and the countries' voting weights, ${ }^{16}$ a probabilistic spatial value would have assigned all the power to the UK, because $\theta$ is fixed. With our specification we are instead able to check the robustness of such assignment and look at how the allocation of power gradually changes by moving away from the symmetric perspective of SSI. We thus find that, when the influence of players' location is present but only moderate, Italy and Spain gain power; while the power of Finland and Portugal increases and remains high by increasing further the coalition-driving role of preferences.

Figure 3 analyzes Germany's power when the proposal coincides with the bliss point of another country - namely, France, Ireland, Portugal, Spain, Sweden and the UK. Germany power is the highest when the proposal is close to Portugal's ideal and lowest when instead is close to that of France. This happens because in the latter case, given the strong similarity in the "idea of Europe" Germany and

\footnotetext{
15 All the calculations were done using Mathematica 6. Code available upon request.

16 The qualified majority threshold was 62 out of 87 and the weights were: France, Germany, Italy, UK 10; Spain 8; Belgium, Greece, Netherlands, Portugal 5; Austria, Sweden 4; Finland, Ireland, Denmark 3; Luxembourg 2.
} 


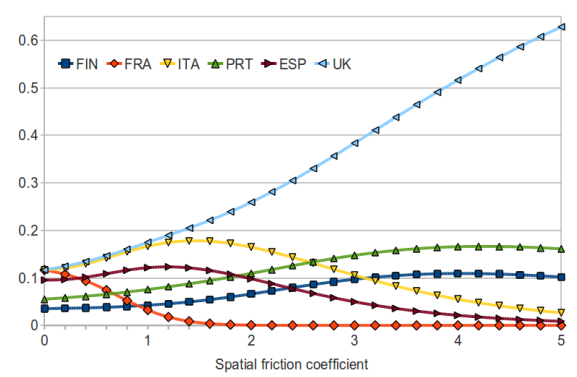

Spatial friction coefficient

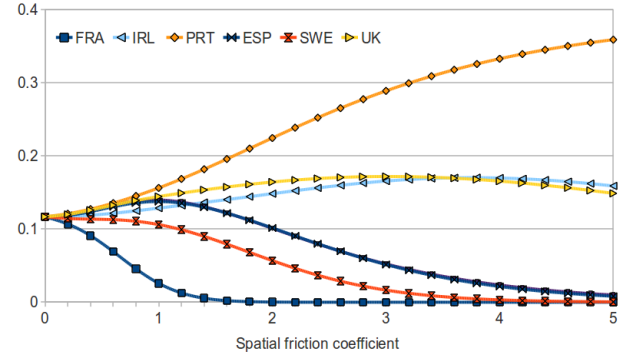

Fig. 2 Pre-Nice power with a constant issue Fig. 3 Pre-Nice Germany's power with issue
$\begin{array}{ll}\text { (Germany's bliss point proposed) } & \text { equal to another country's bliss point }\end{array}$

Fig. 2 Pre-Nice power with a constant issue Fig. 3 Pre-Nice Germany's power witl
$\begin{array}{ll}\text { (Germany's bliss point proposed) } & \text { equal to another country's bliss point }\end{array}$

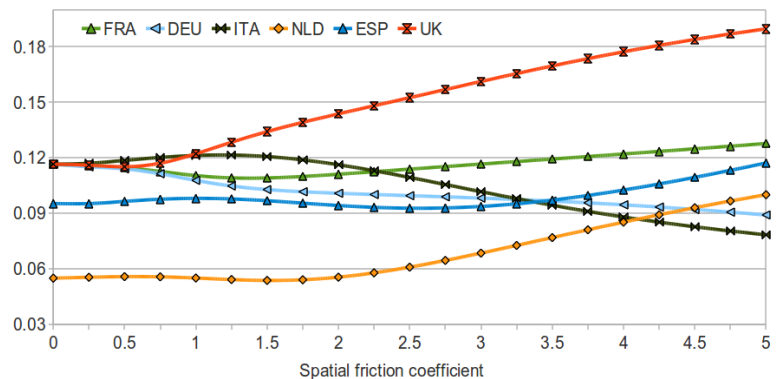

Fig. 4 Pre-Nice power indexes (issues uniformly distributed over countries' bliss points)

France had, Germany is usually an early supporter of French proposals and, as such, not pivotal.

Finally, Figure 4 shows the power indexes in case of probabilistic issues for six of the most powerful countries: France, Germany, Italy, the Netherlands, Spain and the UK. Clearly, the rank correlation between votes and power decreases by increasing the role of preferences in coalition formation. What is less straightforward, power does not necessarily change monotonically with the spatial friction coefficient. Hence, the ranking at one value of $\rho$ is not necessarily a good predictor of the ranking at another value. So, for instance, while a present but mild stickiness of countries at their own ideal points actually increases Italy's power, this decreases when the role of preferences in coalition formation gets stronger. On the contrary, power of the Netherlands is approximately equal to SSI for small $\rho$ but it then increases significantly. The most powerful player in the Pre-Nice scenario instead turns out to be the UK for almost all the range of possible values of $\rho$.

\subsection{EU27 Post-Nice}

The UK is confirmed as the most powerful member in CM also in the EU27 Post-Nice scenario. This results from the interaction between the location of the 


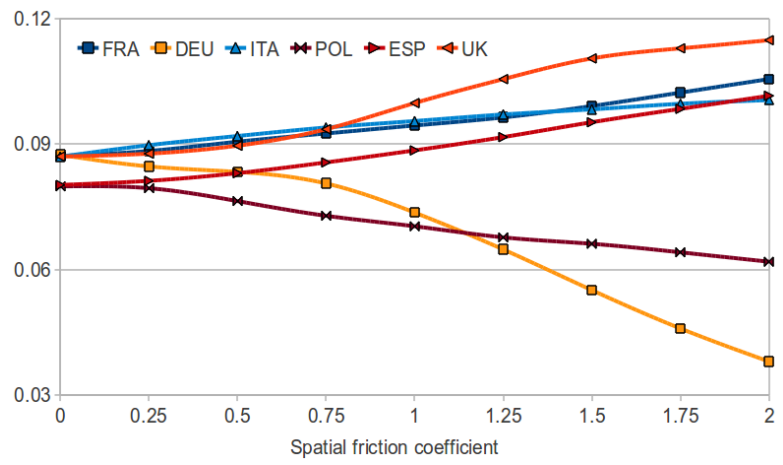

Fig. 5 EU27 Post-Nice power indexes (issues uniformly distributed over countries' bliss points)

27 member states on the ideological space (Figure 1(b)) and the voting weights agreed in the Nice Treaty after the enlargement. ${ }^{17}$

Figure 5 shows the power index of six among the most powerful countries: France, Germany, Italy, Poland, Spain and the UK. The index is computed assuming a uniform discrete distribution of the issue over the bliss points of the 27 member countries and simulating the process of coalition formation (Algorithm 1 in the Appendix) with 5,000,000 repetitions for each $\rho(\Delta \rho=0.25,0 \leq \rho \leq 2)$.

The main result is the striking difference in the dynamics of the index for the UK and Spain, on the one side, and Germany and Poland on the other. Indeed, when the spatial friction coefficient increases, the UK and Spain gain power, while Germany and Poland loose it.

These results are rather different from those obtained by Barr and Passarelli (2009), in spite of the fact that we use their data to locate countries' bliss points. In particular, they find that the UK's power is not that high. Apart from the absence of a random component in the bill-induced ordering, this difference comes also from the assumption they made about the form of the enthusiasm function and the other condition they impose that the bill must lie in the unit sphere. This in turn emphasizes the high dependency of all the non-symmetric extensions of power indexes on the set of assumptions made to identify coalition probabilities.

\footnotetext{
17 The voting system in the Nice Treaty is actually quite complex, as it prescribes three quotas: a) a qualified weighted majority; b) the majority of members; c) $62 \%$ of the total population of the Union. However, as stressed among the others by Felsenthal and Machover (2001) and Baldwin and Widgrén (2004), the latter two conditions have negligible effects on the formation of winning coalitions. As for the first condition, the qualified majority threshold is 255 out of 345 and the countries votes are: France, Germany, Italy, UK 29; Poland, Spain 27; Romania 14; Netherlands 13; Belgium, Czech Rep., Greece, Hungary, Portugal 12; Austria, Bulgaria, Sweden 10; Denmark, Finland, Ireland, Lithuania, Slovakia 7; Cyprus, Estonia, Latvia, Luxembourg, Slovenia 4; Malta 3.
} 


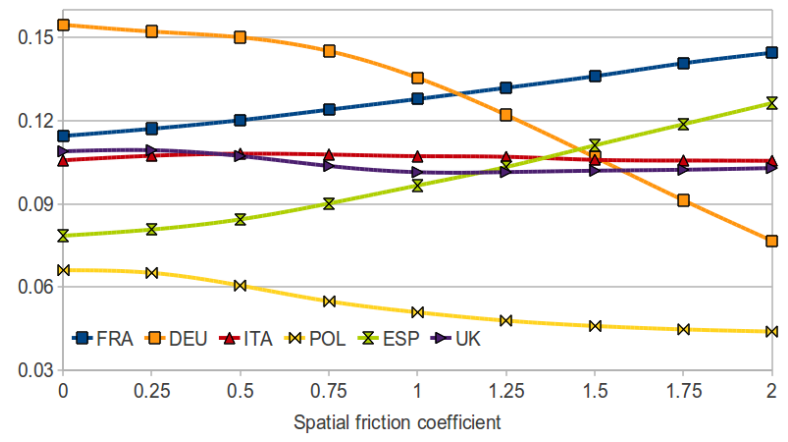

Fig. 6 Lisbon Treaty power indexes (issues uniformly distributed over countries' bliss points)

\subsection{Lisbon Treaty}

Finally, Figure 6 shows the results for the six most powerful countries in the third scenario: 27 member states with the voting rules of the Lisbon Treaty, whose new system is going to come into force in November $2014 .^{18}$

This is substantially a double majority system which prescribes two quotas to pass a bill: i) $55 \%$ of member states, and ii) $65 \%$ of total population. ${ }^{19,20}$

In this case, while for Italy and UK SSI turns out a good predictor of power, even accounting for preferences, differences emerge with respect to the other four countries. Indeed, Germany tends to lose power when countries' stickiness to the ideal points increases, while France and Spain gain it, and France in particular becomes the pivotal player in the majority of coalitions when the spatial friction coefficient is high.

\section{Conclusions}

The analysis of the power distribution entailed by the different voting rules and memberships in the EU have long received attention by researchers, and power indexes have been fruitfully applied in this context.

The symmetry assumption behind the standard indexes, such as the ShapleyShubik index (SSI) and the Penrose-Banzhaf index, implies no role for players' preferences in the process of coalition formation. This assumption can be justified from an a-priori perspective by invoking the principle of insufficient reason and the veil-of-ignorance. Nonetheless, a different perspective can be adopted and it

\footnotetext{
18 Also in this case the index is computed by Monte Carlo simulations $(5,000,000$ repetitions for each $\rho, \Delta \rho=0.25,0 \leq \rho \leq 2)$.

19 We computed percentage population weights from 2010 Eurostat data. They are: Germany 16.32; France 12.91; UK 12.37; Italy 12.04; Spain 9.18; Poland 7.62; Romania 4.28; Netherlands 3.31; Greece 2.26; Belgium 2.16; Portugal 2.12; Czech Rep. 2.10; Hungary 2.00; Sweden 1.86; Austria 1.67; Bulgaria 1.51; Denmark 1.10; Slovakia 1.08; Finland 1.07; Ireland 0.89; Lithuania 0.66; Latvia 0.45; Slovenia 0.41; Estonia 0.27; Cyprus 0.16; Luxembourg 0.10; Malta 0.08.

20 The Lisbon Treaty also provides the possibility for 24 members to jointly pass a proposal, irrespectively of their aggregate population. This has however an overall negligible effect on countries' ability to form winning coalitions. We nevertheless consider such possibility.
} 
is indeed adopted in the theory of spatial indexes, where players' preferences over political outcomes are explicitly accounted for.

In fact, there has been a lively debate on the possibility and utility of introducing preferences in power measurements (e.g. Garrett and Tsebelis 1999b,a; Braham and Holler 2005; Napel and Widgrén 2004, 2005).

Barr and Passarelli (2009) take a pro stance on the issue and apply a generalization of Owen and Shapley's (1989) spatial index to the analysis of the distribution of power within the Council of Ministers (CM).

They observe that the Owen-Shapley index is just a specification of a more general class of indexes, the probabilistic spatial indexes, that calculate power by determining the pivotal players on the base of the preference-induced coalitions conditional on each issue and then randomizing over issues.

Although the approach is appealing and analytically elegant, it suffers from two major drawbacks: i) it produces an excessive concentration of power measures; ii) the indexes are too sensitive to players' locations.

This happens essentially because probabilistic spatial indexes assign zero probability to a huge fraction of possible orderings, since in these indexes the order of players is completely determined once the issue is known. It makes apparent the strong dichotomy that exists between SSI, where all the possible orderings are equiprobable, and probabilistic spatial indexes, where on the contrary there is only one possible order of players consistent with each issue.

In this paper, we have discussed a simple generalization of probabilistic spatial indexes to overcome their main limitations. The main idea is to introduce a random component along with the deterministic one in the process of coalition formation. By calibrating the relative strength of the random component, one is able to produce a continuum of cases ranging from the fully deterministic order (conditional on the issue) of probabilistic spatial indexes and the fully random order of SSI.

The approach is quite general and does not require any particular form of the ordering function. As an illustrative application, we have nevertheless assumed Euclidean preferences of players over political outcomes and reconsidered the analysis of power distribution in CM under three different scenarios: i) Pre-Nice with 15 member states; ii) Post-Nice after the enlargement (27 members); iii) Lisbon Treaty with the present 27 members.

In this application, the UK (France) comes out as the most powerful member state in the Pre-Nice and Post-Nice scenarios (Lisbon Treaty scenario), while Germany's power is lower than that assigned by symmetric indexes of power.

These results are more robust than those obtained from standard spatial analysis, for our approach allows to analyze how they change when the asymmetry assumption is gradually removed. They are nonetheless subject to a number of important caveats - e.g. high dependency on assumptions over preferences and issues; expected discrepancy between Eurobarometer polls and government policy preferences; lack of strategic interactions; etc. - and therefore their importance should not be overstated.

\section{A Algorithms to compute the index}

Here, we present the two algorithms used in the empirical application to compute the indexes.

The first one is a heuristic method which uses the random-order description of PGSV proved in Lemma 2. In particular, the procedure randomly draws the issue from the distribution 
$F_{\theta}$ and for each issue simulates the random sequence of players joining the coalition one by one with the conditional probabilities of equation (15) (Algorithm 1).

In this case, the computation time is decided by the researcher, that fixes the maximum number of simulations. The estimates asymptotically approach the exact values.

As a matter of fact, simulation is essential to get the power index for voting games with continuous distributions of the issues or a large number of players. Indeed, even for a fixed issue, equation (14) must be computed over a number of sequences that is $n$ !. Therefore, the exact computation of the index requires an algorithm with exponential complexity.

This notwithstanding, when the number of players is relatively small, if one assumes a discrete distribution of $\theta$ a more precise algorithm can be implemented which uses the conditional probability of equation (14) (Algorithm 2). ${ }^{21}$ Indeed, in this case, although the computation theoretically requires exponential time, it can be actually completed in a few minutes/hours.
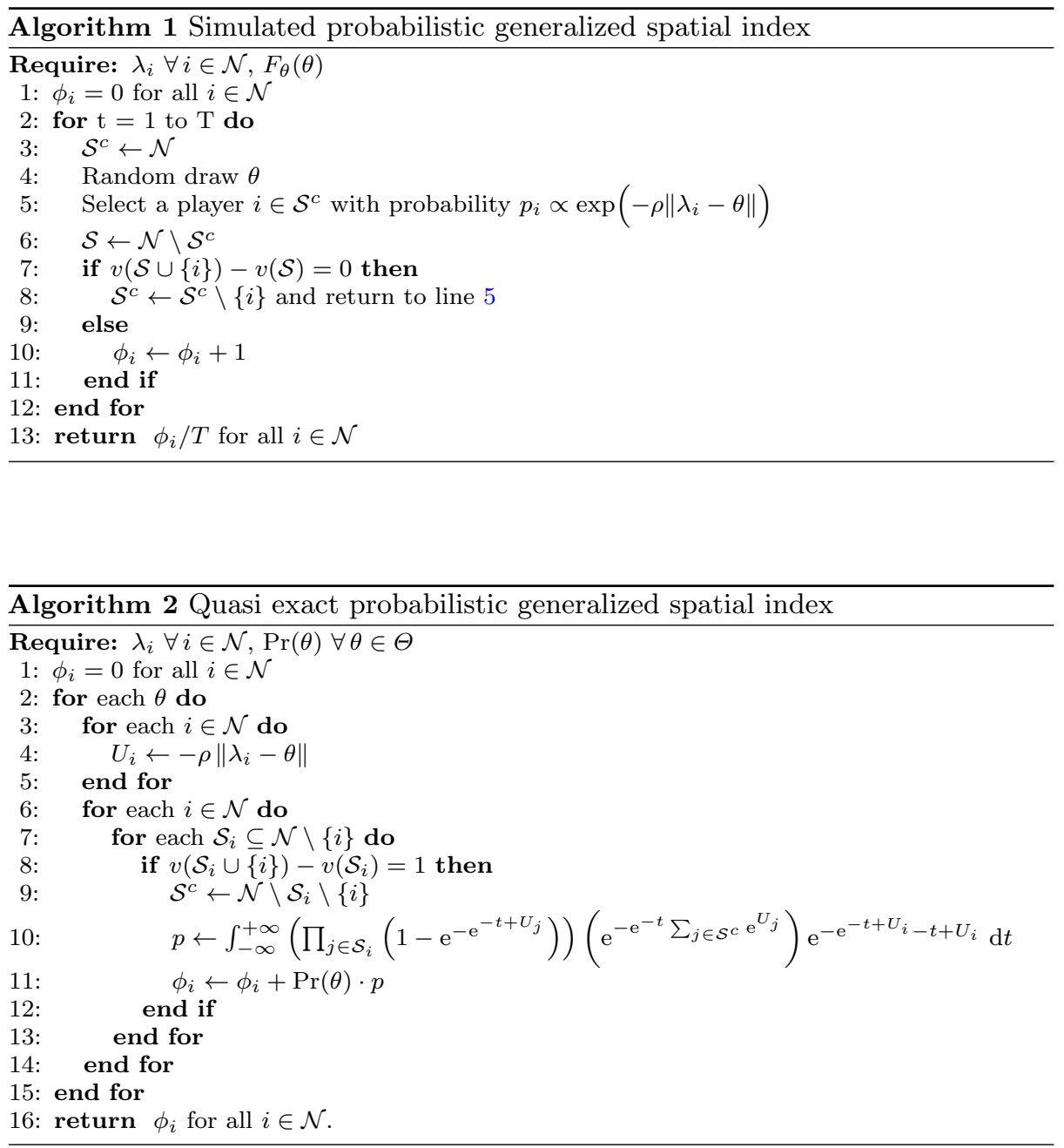

\footnotetext{
21 The algorithm is not exact because it still uses an approximation to calculate the improper
} integral in equation (14). 
Acknowledgements The authors gratefully acknowledge Jason Barr and Francesco Passarelli for the data. In particular, they really appreciate helpful discussions with Francesco Passarelli. They also thank an anonymous referee for very detailed and constructive comments. The usual disclaimer applies.

\section{References}

Baldwin, R. E., and M. Widgrén. 2004. Winners and losers under various dual majority rules for the EU Council of Ministers. In Reasoned choices - Essays in honor of academy Professor Hannu Nurmi on the occasion of his 60th birthday, ed. M. Widgrén. The Finnish Political Science Association.

Banzhaf, J. F. 1965. Weighted voting doesn't work: A mathematical analysis. Rutgers Law Review 19: 317-343.

Barr, J., and F. Passarelli. 2009. Who has the power in the EU? Mathematical Social Sciences 57: 339-366.

Ben-Akiva, M. E., and S. R. Lerman. 1985. Discrete choice analysis: theory and application to travel demand. Cambridge: MIT.

Braham, M., and M. J. Holler. 2005. The impossibility of a preference-based power index. Journal of Theoretical Politics 17: 137-157.

Dubey, P., A. Neyman, and R. J. Weber. 1981. Value theory without efficiency. Mathematics of Operations Research 6: 122-128.

Einy, E., and O. Haimanko. 2011. Characterization of the Shapley-Shubik power index without the efficiency axiom. Games and Economic Behavior forthcoming.

Felsenthal, D. S., and M. Machover. 2001. The treaty of Nice and qualified majority voting. Social Choice and Welfare 18 (3): 431-464.

Felsenthal, D. S., and M. Machover. 2004. Analysis of QM rules in the draft constitution for Europe proposed by the European convention, 2003. Social Choice and Welfare 23 (1): $1-20$.

Garrett, G., and G. Tsebelis. 1999a. More reasons to resist the temptation of power indices in the European Union. Journal of Theoretical Politics 11 (3): 331-338.

Garrett, G., and G. Tsebelis. 1999b. Why resist the temptation to apply power indices to the European Union? Journal of Theoretical Politics 11 (3): 291-308.

Garrett, G., and G. Tsebelis. 2001. Even more reasons to resist the temptation of power indices in the EU. Journal of Theoretical Politics 13 (1): 99.

Humphreys, M., and M. Laver. 2010. Spatial models, cognitive metrics, and majority rule equilibria. British Journal of Political Science 40 (01): 11-30.

Laruelle, A., and F. Valenciano. 2008. Voting and collective decision-making. Cambridge: Cambridge University Press.

McFadden, D. 1984. Econometric analysis of qualitative response models. In Handbook of econometrics, eds. Z. Griliches and M. D. Intriligator, Vol. II. Elsevier.

Milyo, J. 2000. Logical deficiencies in spatial models: A constructive critique. Public Choice 105: 273-289.

Monderer, D., and D. Samet. 2002. Variations on the Shapley value. In Handbook of game theory, eds. R. J. Aumann and S. Hart, Vol. III. Amsterdam: Elsevier Science.

Napel, S., and M. Widgrén. 2004. Power measurement as sensitivity analysis. A unified approach. Journal of Theoretical Politics 16 (4): 517-538.

Napel, S., and M. Widgrén. 2005. The possibility of a preference-based power index. Journal of Theoretical Politics 17 (3): 377-387.

Napel, S., and M. Widgrén. 2006. The inter-institutional distribution of power in EU codecision. Social Choice and Welfare 27 (1): 129-154.

Napel, S., and M. Widgrén. 2008. Shapley-Shubik versus strategic power - Live from the UN Security Council. In Power, freedom and voting - Essays in honor of Manfred J. Holler, eds. M. Braham and F. Steffen, 99-118. Berlin: Springer.

Napel, S., and M. Widgrén. 2011. Strategic versus non-strategic voting power in the EU Council of Ministers: the consultation procedure. Social Choice and Welfare forthcoming: 1-31.

Owen, G. 1971. Political games. Naval Research Logistics Quarterly 18 (3): 345-355.

Owen, G., and L. S. Shapley. 1989. Optimal location of candidates in ideological space. International Journal of Game Theory 18: 339-356. 
Passarelli, F., and J. Barr. 2007. Preferences, the agenda setter, and the distribution of power in the EU. Social Choice and Welfare 28 (1): 41-60.

Penrose, L. S. 1946. The elementary statistics of majority voting. Journal of the Royal Statistical Society 109 (1): 53-57.

Roth, A. E., ed. 1988. The Shapley value. Essays in honor of Looyd S. Shapley. Cambridge: Cambridge University Press.

Shapley, L. S. 1953. A value for n-person games. In Contributions to the theory of games, eds. H. W. Khun and A. W. Tucker, Vol. II, 307-317. Princeton: Princeton University Press.

Shapley, L. S., and M. Shubik. 1954. A method for evaluating the distribution of power in a committee system. American Political Science Review 48: 787-792.

Shepard, R. N. 1987. Toward a universal law of generalization for psychological science. Science 237 (4820): 1317.

Steunenberg, B., D. Schmidtchen, and C. Koboldt. 1999. Strategic power in the European Union. Journal of Theoretical Politics 11 (3): 339-366.

Straffin, P. D. 1977. Homogeneity, independence, and power indices. Public Choice 30: 107-118.

Walker, J., and M. Ben-Akiva. 2002. Generalized random utility model. Mathematical Social Sciences 43 (3): 303-343.

Weber, R. J. 1988. Probabilistic values for games. In The Shapley value: Essays in honor of Lloyd S. Shapley, ed. A. E. Roth, 101-119. Cambridge: Cambridge University Press. 